Cahiers de la recherche sur les droits fondamentaux

Surveiller et punir / Surveiller ou punir?

\title{
Avant-propos sur l'inscription des droits sociaux dans la Convention européenne des droits de l'homme
}

Préalables méthodologiques sur la justiciabilité des droits sociaux

Jean-François Akandji-Kombé

\section{OpenEdition \\ Journals}

Édition électronique

URL : https://journals.openedition.org/crdf/7536

DOI : $10.4000 /$ crdf.7536

ISSN : 2264-1246

Éditeur

Presses universitaires de Caen

Édition imprimée

Date de publication : 1 octobre 2004

Pagination : 83-92

ISBN : 2-84133-221-7

ISSN : $1634-8842$

Référence électronique

Jean-François Akandji-Kombé, « Avant-propos sur l'inscription des droits sociaux dans la Convention européenne des droits de l'homme », Cahiers de la recherche sur les droits fondamentaux [En ligne], 3। 2004, mis en ligne le 18 décembre 2020, consulté le 14 novembre 2022. URL : http://

journals.openedition.org/crdf/7536; DOI : https://doi.org/10.4000/crdf.7536 


\title{
Avant-propos sur l'inscription des droits sociaux dans la Convention européenne des droits de l'homme
}

\section{Préalables méthodologiques sur la justiciabilité des droits sociaux}

\author{
Jean-François AKANDJI-KOMBÉ \\ Maître de conférences à I'Université de Caen Basse-Normandie \\ Responsable du Master "Contentieux et pratique des droits fondamentaux "
}

I. Première proposition : la thèse de la non-justiciabilité des droits sociaux est fondée en grande partie sur une erreur d'appréciation, sur une confusion entre technique de formulation et substance des droits

II. Seconde proposition : la protection juridictionnelle de tous les droits sociaux est concevable si l'on admet qu'une telle protection ne se limite pas à la mise en œuvre judiciaire de droits directement applicables

III. Troisième proposition : en définitive les exigences des droits sociaux - dont dépend aussi la nature du contrôle qui sera mis en œuvre - n'apparaissent pas fondamentalement différentes de celles des droits civils et politiques

Les droits sociaux n'ont en général pas bonne presse. Le juriste hésite volontiers entre condescendance et suspicion : condescendance à l'égard d'une catégorie mineure dans le champ du droit des droits de l'homme; suspicion à l'égard d'un ensemble ayant tout du cheval de Troie, venant prétendument subvertir la problématique des droits de l'homme et faisant courir aux libertés dites classiques un risque majeur: celui de la dilution. L'observateur quant à lui a pris l'habitude du constat désabusé en voyant fonctionner les mécanismes consacrés à la garantie de ces droits et en les comparant à ceux destinés à la protection des droits civils et politiques.
Les formules employées s'agissant des instruments du Conseil de l'Europe et des droits protégés par eux sont aussi instructives que célèbres. Qui ne connaît le titre interrogatif, si évocateur, de l'article de M. Pierre-Henri Imbert: "Droits des pauvres, pauvre(s) $\operatorname{droit}(s) »{ }^{1}$, ou encore l'affirmation de $\mathrm{M}^{\mathrm{me}}$ Catherine Lalumière, alors Secrétaire général du Conseil de l'Europe, selon laquelle la Charte sociale européenne «fait figure de parent pauvre voire de tigre de papier ${ }^{2}$ comparée à la Convention européenne des droits de l'homme? Et d'autres auteurs encore de conclure à une « existence crépusculaire » de la Charte $^{3}$. Ces formules sont, il faut bien l'admettre, plus

1. P.-H. Imbert, «Droits des pauvres, pauvre(s) droit(s) ? Réflexion sur les droits économiques, sociaux et culturels », Revue de droit public, 1989, p. 740. P.-H. Imbert est Directeur général des droits de l'homme du Conseil de l'Europe.

2. J. Dhommeaux, «La contribution du Comité des droits économiques, sociaux et culturels des Nations Unies à la protection des droits économiques, sociaux et culturels ", AFDI, 1994, p. 635.

3. D. Gomien, D. Harris et L. Zwaak, Convention européenne des doits de l'homme et Charte sociale européenne, droit et pratique, Strasbourg, Éditions du Conseil de l'Europe, 1997, p. 402. 
que des jeux de mots ou des traits d'esprit. Elles dépeignent une situation qui a pu faire craindre que l'incorporation des droits sociaux dans la Convention vienne saper la crédibilité de cette dernière et, partant, son efficacité.

Cette crainte sous-tend les grands textes du Conseil de l'Europe, y compris ceux qui, ces dernières années, s'occupent de tracer les grandes lignes de l'évolution de cette organisation, notamment sur le plan normatif. Sans doute y trouve-t-on l'affirmation de l'impératif d'une meilleure protection des droits sociaux. Mais outre que cette affirmation est souvent marginale dans l'économie générale de ces documents, l'incorporation des droits sociaux à la Convention EDH est une hypothèse ignorée par les représentants gouvernementaux, sans doute de propos délibéré. Les projections d'avenir en matière de protection des droits de l'homme se départissent ainsi à grand-peine d'un schéma dual, voire binaire, largement enraciné dans le passé, faisant reposer cette protection d'un côté sur la Convention EDH et de l'autre sur la Charte sociale européenne.

Tout appelle pourtant, et ce sera vraisemblablement de plus en plus le cas, à son dépassement, à commencer par le renouveau de la doctrine de l'indivisibilité des droits de l'homme. Renouveau parce que cette doctrine n'est précisément pas nouvelle. On pourrait même dire qu'elle est la matrice du droit international contemporain des droits de l'homme dans la mesure où celui-ci puise sa source et trouve son fondement dans la Déclaration universelle des droits de l'homme. Du moins en est-il ainsi si l'on retient la vision unitaire - et largement idéalisée qu'en a développé René Cassin dans le cours qu'il professa à l'Académie de droit international de La Haye. Rappelons que selon la présentation de l'illustre auteur, la Déclaration figurerait le vaste portique d'un temple dont le parvis serait formé par le préambule, soutenu par quatre colonnes dont celle des droits économiques, sociaux et culturels, colonne dont il affirme la symétrie avec celle des droits et libertés d'ordre personnel, objets des articles 3 à 11, l'ensemble marquant « un élan continu de l'individuel vers le social ${ }^{4}$. On le sait, ce n'est pas cette vision qui a finalement triomphé lors de l'élaboration des Pactes ${ }^{5}$.

Mais s'il faut bien parler de renouveau, c'est aussi parce que la doctrine de l'indivisibilité des droits de l'homme a gagné du terrain, passant de l'affirmation doctrinale à une expression politique qui annonce ou, plus précisément, appelle la concrétisation juridique. C'est dans cette perspective que se situe, parmi d'autres textes de même nature, la déclaration adoptée par le Comité des ministres du Conseil de l'Europe à l'occasion du cinquantième anniversaire de la Déclaration universelle des droits de l'homme, le 10 décembre 1998, laquelle s'inspire d'ailleurs largement de la déclaration de la Conférence mondiale sur les droits de l'homme de Vienne de 1993. Les gouvernements des États membres y «réaffirment que tous les droits de l'homme sont universels, indissociables, interdépendants et intimement liés, qu'ils soient civils, politiques, économiques, sociaux ou culturels", ainsi que «le besoin de renforcer les droits économiques et sociaux fondamentaux». La Conférence ministérielle de Rome sur les droits de l'homme (3-4 novembre 2000) n'a pas manqué à son tour de rappeler cette indivisibilité et cette interdépendance des droits de l'homme ${ }^{6}$. Et s'il est vrai qu'un fossé sépare encore à l'heure actuelle le discours de la pratique, le défi semble avoir définitivement changé d'objet : il s'agit dorénavant de briser le mur de ce que le Secrétaire général du Conseil de l'Europe, M. Walter Schwimmer, a appelé "l'invisibilité de l'indivisibilité » ${ }^{7}$, c'est-à-dire de réconcilier discours et pratique en inscrivant l'indivisibilité dans les faits, ce qui suppose qu'elle s'inscrive d'abord dans l'ordre du droit.

D’ores et déjà, le mouvement est amorcé. Au conseil de l'Europe: d'abord avec le rapprochement du régime de protection des droits sociaux de celui des droits civils et politiques impliqué par la relance de la Charte sociale européenne et, surtout, par l'adoption et la mise en œuvre du protocole de 1995 sur les réclamations collectives ${ }^{8}$; ensuite par le jeu probable du principe général de nondiscrimination énoncé par le protocole $\mathrm{n}^{\mathrm{o}} 12$ à la Convention $\mathrm{EDH}$, lorsque cet instrument, ouvert à ratification le 4 novembre 2000, entrera en vigueur.

Les progrès sont tout aussi, sinon plus significatifs encore dans d'autres cadres, notamment internationaux. On peut ainsi mentionner:

- l'adoption en 1988 et l'entrée en vigueur en 1999 d'un protocole additionnel à la Convention américaine relative aux droits de l'homme, dit "protocole de San Salvador ", lequel est consacré aux droits économiques, sociaux et culturels et prévoit la possibilité de recours proprement juridictionnels pour la sauvegarde du droit syndical et du droit à l'éducation ${ }^{9}$;

4. RCADI, 1951-II, p. 277-279.

5. Il est intéressant de noter que cette approche unitaire présida pendant un temps aux travaux de la Commission des droits de l'homme. Les directives de l'Assemblée générale des Nations Unies contenues dans la résolution 421 (V) 1950 étaient claires à cet égard. Convaincue que «la jouissance des libertés civiques et politiques et celle des droits économiques, sociaux et culturels sont liées entre elles et se conditionnent mutuellement », l'Assemblée avait demandé à la Commission des droits de l'homme de préparer un texte unique qui engloberait l'ensemble des droits de l'homme. La solution de l'élaboration de deux instruments distincts s'est toutefois imposée rapidement, dès 1952, après les débats longs et quelque peu vifs qui ont eu lieu au sein de la Commission au cours de ses réunions en 1951-1952. Cette solution est exprimée par la résolution 543 (VI) 1950. Voir notamment Imre Szabo, "Historical foundations of Human Rights and subsequent developments », in K. Vasak (édité en anglais par P. Alston), The International Dimensions of Human Rights, vol. 1, Westport, Greenwood Press et Paris, UNESCO, 1982, p. 11 sq.

6. Document CM (2000) du 14 novembre 2000

7. Ibid., p. 27.

8. Voir notamment J.-F. Akandji-Kombé, «L'application de la Charte sociale européenne: la mise en œuvre de la procédure de réclamations collectives», Droit social, $\mathrm{n}^{\circ}$ 9-10, 2000, p. 888 sq. Sur les développements de la procédure, voir notre chronique annuelle dans la $R T D H$.

9. Aux termes de l'article $19(6)$ : «Au cas où [ces droits] ont été violés par une action imputable directement à un État partie au présent protocole, cette situation peut donner lieu, par le recours à la Commission interaméricaine des droits de l'homme et, le cas échéant, à la Cour interaméricaine des 
- l'adoption par le Conseil européen de Nice, en décembre 2000, d'une Charte des droits fondamentaux de l'Union européenne ${ }^{10}$ qui, abstraction faite de son absence actuelle de normativité, présente au moins deux intérêts pour le présent propos: la réunion des droits civils, politiques, économiques et sociaux dans un même document d'une part ; d'autre part l'affirmation du principe d'une protection juridictionnelle globale des droits énoncés, sous réserve d'une différenciation des modalités de protection dont la portée reste à déterminer ;

- l'élaboration, en cours, d'un protocole facultatif se rapportant au Pacte international relatif aux droits économiques, sociaux et culturels, instituant une procédure de réclamations individuelles, inspiré du protocole attaché au Pacte relatif aux droits civils et politiques ${ }^{11}$.

Ces différents textes et initiatives attestent d'un mouvement général de renforcement de la protection des droits économiques, sociaux et culturels, et traduisent par conséquent une tendance profonde à l'unification du régime juridique des droits de l'homme pris dans leur ensemble, s'agissant à tout le moins de ceux qui relèvent des deux premières "générations ». On observera par ailleurs qu’ils placent le système européen des droits de l'homme - celui du Conseil de l'Europe - dans une position plutôt incongrue, celle d'un système en voie de dépassement.

Il y a là une incitation forte au perfectionnement du dispositif européen. Ce n'est dès lors pas un hasard si, ces dernières années, sont apparues diverses initiatives institutionnelles tendant à réaliser l'effectivité des droits sociaux. Ce fut d'abord, comme on l'a vu, la rénovation du système de la Charte sociale européenne, concrétisée par l'adoption de différents protocoles additionnels entre 1988 et 1995 et par l'adoption d'une Charte révisée en 1996. Mais ce sont aussi des projets plus ambitieux, visant à l'unification des droits de l'homme et du système européen de protection par l'inscription des droits sociaux dans le système de la Convention EDH. L'Assemblée parlementaire du Conseil de l'Europe s'est prononcée en ce sens à plusieurs reprises, et notamment dans un texte adopté le 23 juin $1999^{12}$, par lequel elle recommandait au Comité des ministres l'élaboration d'un protocole additionnel à la Convention EDH garantissant les droits sociaux. La protection des droits sociaux dans le cadre même de la Convention EDH apparaît ainsi comme un défi lancé au Conseil de l'Europe à l'aube de ce troisième millénaire.

Il importe immédiatement de préciser que cette circonstance ne signifie nullement que les droits sociaux sont absents du dispositif actuel de la Convention. On sait que celle-ci offre une protection directe à un nombre limité de droits de ce type: ainsi de la liberté syndicale (art.11) et de la liberté professionnelle (art. 4), laquelle est formulée de manière négative à travers la prohibition du travail forcé ou obligatoire ${ }^{13}$. Par ailleurs et de manière plus significative, la matière sociale a progressivement, sous l'impulsion de la jurisprudence, investi le champ de la Convention en donnant une certaine consistance à l'idée, exprimée par la Cour EDH dans l'arrêt Airey du 9 octobre 1979, selon laquelle «nulle cloison étanche » ne sépare les droits économiques et sociaux des droits civils et politiques. Mais précisément, comme l'ont montré les études consacrées à cette évolution ${ }^{14}$, les droits sociaux ne sont pas pour autant entrés de plain-pied dans le système conventionnel. Pour reprendre une formule jurisprudentielle récurrente, ils ne sont pas protégés « en tant que tels ", mais seulement à titre de prolongement des droits civils et politiques. Il n'en demeure pas moins que l'intégration des droits sociaux dans le système de la Convention EDH se présente, du fait même de cette jurisprudence, moins comme un appel à l'inédit que comme une exigence d'approfondissement à partir de prémisses d'ores et déjà présentes dans le système actuel. Sa réalisation pourrait aussi être souhaitée à titre de clarification, dans la mesure où les lacunes actuelles pourraient entraîner des audaces interprétatives susceptibles d'affecter la crédibilité de la jurisprudence par défaut de consensus des États sur les valeurs et les droits ainsi protégés.

Reste que la perspective d'une incorporation des droits sociaux à la Convention ne va pas de soi. Elle suppose que soient levés des obstacles politiques, mais surtout que soient résolues certaines difficultés juridiques. Les unes sont de principe et concernent principalement le statut des droits sociaux par rapport aux droits civils et politiques, la question étant ici de savoir si les droits de la

droits de l'homme, à l'application du système de requêtes individuelles prévues aux articles 44 à 51 et 61 à 69 de la Convention américaine relative aux droits de l'homme.»

10. Voir en particulier La Charte des droits fondamentaux de l'Union européenne, son apport à la protection des droits de l'homme en Europe, J.-Y. Carlier et O. De Schutter (dir.), Bruxelles, Bruylant, 2002; F. Benoît-Rohmer, «La Charte des droits fondamentaux de l'Union européenne ou une remise en cause des poncifs en matière de droits économiques et sociaux", in Les Droits sociaux ou la démolition de quelques poncifs, C. Grewe et F. Benoît-Rohmer (dir.), Strasbourg, Presses universitaires de Strasbourg, 2003, p. 169 sq.

11. Voir notamment G. Malinverni, «Le projet de protocole additionnel au Pacte international relatif aux droits économiques, sociaux et culturels», in Les Droits sociaux..., p. 95 sq.

12. Recommandation $n^{\circ} 1415$ (1999) de l'Assemblée parlementaire en date du 23 juin 1999. Sur ce texte, voir le rapport présenté au nom de la Commission des questions sociales, de la santé et de la famille par $\mathrm{M}^{\mathrm{me}}$ Pilar Pulgar (Doc. 8357 du 23 mars 1999) et l'avis de la Commission des questions juridiques et des droits de l'homme, rapporté par M. Erik Jurgens (Doc 8433 du 2 juin 1999). Il est à noter que l'incorporation des droits sociaux à la Convention $\mathrm{EDH}$ avait déjà fait l'objet de la recommandation n 838 (1978) de l'Assemblée parlementaire. Elle fut à nouveau envisagée au milieu des années 80 comme une alternative sérieuse à la relance de la Charte sociale européenne (voir spécialement l'avis n 127 (1986) de l'Assemblée «sur le projet de troisième plan à moyen terme (1987-1991) du Conseil de l'Europe», point 10, C, c).

13. On ne retient ici que les droits proprement sociaux à l'exclusion de ceux qui comportent une dimension sociale tout en étant traditionnellement considérés comme des droits civils. Pour un recensement exhaustif appuyé sur une telle approche, voir M. Enrich Mas, «Les droits sociaux dans la jurisprudence de la Cour et de la Commission européennes des droits de l'homme», RTDH, 1992, p. 147 sq.

14. Voir en particulier F. Sudre, "La perméabilité de la Convention européenne des droits de l'homme aux droits sociaux", in Mélanges J. Mourgeon, Bruxelles, Bruylant, 1998, p. 467. 
première catégorie, pris dans leur ensemble ou isolément, sont susceptibles de protection juridictionnelle. Dans l'affirmative, d'autres difficultés surgissent qui se rapportent plutôt à la mise en œuvre de la protection juridictionnelle : quelles pourraient en être les modalités et comment articuler cette garantie juridictionnelle et les mécanismes et procédures d'un autre type existant actuellement sur le fondement d'autres instruments telle la Charte sociale européenne, si tant est que de tels mécanismes puissent ou doivent survivre à la réalisation du projet évoqué plus haut? Bref, envisager la protection des droits sociaux dans le cadre de la Convention EDH supposerait au fond que l'on réponde à la double question des droits à protéger et de la protection à mettre en œuvre.

On a pris le parti ici de ne pas aborder cet aspect-ci de la question. Étant donné l'état embryonnaire des discussions en la matière, l'on peut se permettre de renvoyer à plus tard l'analyse des données techniques et des implications pratiques d'une telle réforme. Avant tout, il importe de répondre à la question de principe. On le fera en partant de trois propositions simples qui tendront à montrer que la "présomption d'injusticiabilité » ${ }^{15}$ qui frappe cette catégorie de droits est parfaitement réfragable. Il doit être précisé d'emblée que ces propositions, tout en s'appuyant sur des arguments tirés du droit positif interne ou international, ne se veulent pas un reflet exact de celui-ci. La raison en est à la fois pratique et théorique. En effet, outre qu'il n'y a pas suffisamment de place dans un exercice comme celui-ci pour un compte rendu exhaustif ou seulement significatif des éléments de droit national accréditant la thèse de la justiciabilité des droits sociaux, on doit aussi tenir compte de la contingence même de ce droit positif, de sa variabilité dans le temps et dans l'espace. Bref, la position fondamentale adoptée à travers les propositions qui suivent est de ne pas inférer l'absence de justiciabilité des droits sociaux simplement du fait que des ordres juridiques, fussent-ils majoritaires, en disposent ainsi sur la base d'arguments et de postulats qui, la plupart du temps, n'ont rien de juridiquement nécessaire.

\section{Première proposition : la thèse de la non-justiciabilité des droits sociaux est fondée en grande partie sur une erreur d'appréciation, sur une confu- sion entre technique de formulation et substance des droits}

Il suffit pour s'en convaincre de rappeler le raisonnement qui sous-tend cette thèse. Fondamentalement, l'absence de justiciabilité des droits sociaux est présentée comme la conséquence directe d'une différence de nature de ces droits d'avec les droits civils et politiques. Or, de quoi déduit-on cette nature? Principalement de la forme en laquelle ces droits sont proclamés par les conventions internationales ou par les constitutions internes.

S'agissant des traités internationaux, on fait d'abord observer que ces droits font l'objet d'actes conventionnels autonomes par rapport à ceux consacrés aux droits civils et politiques, ce qui est objectivement le cas sur le plan mondial avec les Pactes et sur le plan européen avec la Convention européenne des droits de l'homme et la Charte sociale européenne. On constate ensuite que ces « droits » sont formulés essentiellement en termes d'obligations juridiques pour les États et que ces obligations sont conçues de manière si souple qu'elles s'apparentent dans la plupart des cas à de simples objectifs. Et pour couronner l'ensemble, on insiste sur le fait, incontestable d'ailleurs, que la garantie des droits sociaux est conçue de manière différente par rapport à celle des droits civils et politiques, et qu'en particulier les mécanismes et procédures de sauvegarde sont plus souples et excluent tout contrôle juridictionnel. Le raisonnement peut devenir plus subtil encore, chacun de ces éléments étant présenté comme la conséquence nécessaire d'une prétendue nature des droits sociaux dont on serait bien en peine de dire l'origine et la teneur.

En revenant à l'argumentation principale, il n'échappera à personne qu'aucun des éléments précédemment rappelés n'est de nature à établir une quelconque différence de nature des droits sociaux. Ces éléments n'ont au demeurant rien de nécessaire juridiquement parlant. Sans doute la différenciation des droits du point de vue de leur consécration et de leur régime est un fait indiscutable qui produit des conséquences juridiques tout aussi incontestables. Mais il faut bien avoir conscience du fait que cette solution de la différenciation a été politiquement voulue, préférée à d'autres solutions, et cela pour des raisons diverses, dont certaines peuvent être de pure circonstance.

Il en a été ainsi de la décision de rédiger deux pactes, ou de celle de cantonner la Convention EDH aux droits civils et politiques en renvoyant à un autre instrument la reconnaissance des droits économiques et sociaux. Il en a été de même s'agissant de la rédaction des dispositions des conventions considérées et, en particulier, de celles du Conseil de l'Europe. À cet égard, la lecture des travaux préparatoires de la Charte sociale européenne montre clairement que les instances d'élaboration ont longtemps hésité entre une rédaction "à la $\mathrm{CEDH}$ », techniquement possible, et les formulations plus souples qui ont finalement été retenues ${ }^{16}$.

Enfin, s'agissant de la garantie de ces droits, on doit admettre que l'exclusion du contrôle juridictionnel est souvent justifiée, de la part des États eux-mêmes, par la

15. L'expression est du Professeur F. Sudre, «Le protocole additionnel à la Charte sociale européenne prévoyant un système de réclamations collectives », RGDIP, 1996-3, p. 739.

16. Voir à ce propos l'éclairante analyse de J.-M. Larralde, « Charte sociale et Convention européenne des droits de l'homme», in La Charte sociale européenne (actes des $1{ }^{\text {res }}$ Rencontres européennes de Caen, 17 mars 200o), J.-F. Akandji-Kombé et S. Leclerc (éd.), Bruxelles, Bruylant (Rencontres européennes), 2001, p. 123 sq. 
nature des droits en cause. Mais là encore, le fait que les objections des États soient formulées en termes juridiques et justifiées juridiquement ne doit pas abuser. L'expérience en cours d'élaboration d'un protocole additionnel au Pacte international sur les droits économiques, sociaux et culturels montre que les arguments juridiques s'inscrivent dans une stratégie d'ensemble dont les ressorts se situent ailleurs, qu'ils sont susceptibles d'évoluer ou de varier, bref ils attestent d'une instrumentalisation du droit qui interdit d'apporter un crédit exagéré aux arguments juridiques avancés ${ }^{17}$.

Il convient d'ailleurs de ne pas perdre de vue que l'exclusion du contrôle juridictionnel par les instruments internationaux de garantie des droits sociaux ne vaut en règle générale que pour la dimension internationale de cette garantie. Une telle exclusion ne fait pas obstacle au contrôle juridictionnel national de l'application et du respect de l'instrument considéré, pas plus qu'elle n'interdit au juge interne d'accorder le bénéfice de l'effet direct à une quelconque des normes édictées par les instruments de garantie des droits sociaux, ou d'en admettre l'invocabilité juridictionnelle. On peut le vérifier tant à propos du Pacte international relatif aux droits économiques, sociaux et culturels qu'avec la Charte sociale européenne.

S’agissant du premier instrument, on tire généralement de la comparaison de son article 2 et de celui du Pacte sur les droits civils et politiques la conclusion que les dispositions du premier texte ne peuvent donner lieu à application juridictionnelle au plan interne. En effet, alors que dans le Pacte sur les droits civils et politiques les États «s'engagent à respecter et à garantir à tous les individus se trouvant sur leur territoire et relevant de leur compétence les droits reconnus [...] sans distinction aucune $[\ldots]$ » et, surtout, «à garantir que toute personne dont les droits et libertés sont reconnus auront été violés disposera d'un recours juridictionnel utile [...] », ils ne s'engagent dans le Pacte relatif aux droits économiques, sociaux et culturels qu' « à agir [...] au maximum de [leurs] ressources disponibles en vue d'assurer progressivement le plein exercice des droits reconnus dans le présent Pacte par tous les moyens appropriés, y compris en particulier l'adoption de mesures législatives». La différence de rédaction, voire de structure des obligations, est patente. Elle n'emporte toutefois pas les conséquences radicales que l'on se plaît parfois à souligner, notamment s'agissant des possibilités de protection juridictionnelle interne. Le Comité des droits économiques, sociaux et culturels a eu le mérite de le rappeler dans ses observations générales relatives à l'application du Pacte correspondant. Ainsi, dans l'observation générale $n^{\circ} 3$ formulée en 1990 concernant la nature des obligations des États parties en vertu de l'article 2, par. 1 du PIDESC ${ }^{18}$, il souligne que «parmi les mesures qui pourraient être considérées comme "appropriées" figurent, outre les mesures législatives, celles qui prévoient des recours judiciaires au sujet de droits qui, selon le système juridique national, sont considérés comme pouvant être invoqués devant les tribunaux ${ }^{19}$. C'est la même analyse qui est développée en 1998 dans l'observation générale $n^{\circ} 9$ relative à l'application du PIDESC au niveau national ${ }^{20}$. On notera en particulier que, pour le Comité, le fait que le PIDESC ne contienne pas, à l'instar du Pacte relatif aux droits civils et politiques, de dispositions obligeant les États parties à garantir le droit à un recours juridictionnel aux victimes de violations des droits protégés, ne signifie pas nécessairement que ces États puissent en toute discrétion exclure de telles possibilités de recours. L'absence de voies de recours juridictionnels pour la sanction des violations des droits économiques, sociaux et culturels devra toujours être justifiée par l'État: celui-ci «devra montrer soit que de tels recours ne constituent pas des "moyens appropriés" au sens du paragraphe 1 de l'article $2 \mathrm{du}$ Pacte relatif aux droits économiques, sociaux et culturels, ou qu'ils sont, compte tenu des autres moyens utilisés, superflus ${ }^{21}$. De telles situations devraient rester exceptionnelles car, de l'avis du Comité, «dans bien des cas, les autres moyens utilisés risquent d'être inopérants s'ils ne sont pas renforcés ou complétés par des recours juridictionnels ${ }^{22}$.

17. On lira avec intérêt les observations faites par les États à propos du projet de protocole additionnel: notamment les rapports du Comité économique et social des Nations Unies nº E / CN.4 / 1998 / 84 du 16 janvier 1998; E / CN.4 / 1998 / 84 / Add. 1 du 16 mars 1998; E/ CN.4 / 1999 / 112 du 7 janvier 1999. Les États opposés à l'adoption du protocole tiennent volontiers pour acquis que les droits sociaux ne peuvent faire l'objet d'une application juridictionnelle et que leur mise en œuvre « dépend du contexte économique, social et culturel issu des politiques générales des divers pays, ainsi que des conditions extérieures, et ne peut souvent pas être assurée par le biais de la législation nationale» (Allemagne). Pour illustrer le propos, ils citent immanquablement le droit au travail, lequel apporterait la preuve de l'impossibilité d'une jouissance immédiate des droits de cette catégorie, puisqu'aucun État n'est en mesure d'éliminer le chômage (Allemagne et Canada en particulier).

18. Voir Récapitulation des observations générales ou recommandations générales adoptées par les organes créés en vertu d’instruments internationaux relatifs aux droits de l'homme, Doc $\mathrm{n}^{\circ} \mathrm{HRI} / \mathrm{GEN} / 1 /$ Rev. 4, février 2000, p. 10.

19. Plus précisément, «Le Comité note, par exemple, que la jouissance des droits reconnus, sans discrimination, est souvent réalisée de manière appropriée, en partie grâce au fait qu'il existe des recours judiciaires ou d'autres recours utiles. [...] En outre, il y a [selon lui] dans le Pacte international relatif aux droits économiques, sociaux et culturels un certain nombre de dispositions qui, semble-t-il, sont susceptibles d'être immédiatement appliquées ». Le Comité range dans cette catégorie notamment les articles 3 [non-discrimination entre hommes et femmes dans la jouissance des droits consacrés], 7 al. a, i [droit à un salaire équitable], 8 [droit syndical et droit de grève], $10 \$_{3}$ [droit des enfants et adolescents à la protection et à l'assistance sans discrimination], $13 \$ 2$ al. a, $\$ 3$ et 4 [caractère obligatoire et gratuité de l'enseignement primaire; droit des parents au libre choix du système d'éducation; principe de libre création et de libre direction des établissements d'enseignement], art. $15 \$ 3$ [liberté de la recherche scientifique et des activités créatrices]. Mieux encore, dans tous les cas où des mesures spécifiques visant à assurer l'exercice des droits reconnus par le Pacte ont été adoptées sous forme législative ou à chaque fois que les dispositions du Pacte ont été incorporées au droit national, le Comité entend obtenir des États qu'ils lui précisent si ces dispositions sont assorties de voies de recours devant le juge.

20. Voir Récapitulation des observations générales..., p. 55.

21. Ibid., p. 56 .

22. Ibid. 
En ce qui concerne la Charte sociale européenne, on sait que la partie III de son annexe « contient des engagements juridiques de caractère international dont l'application est soumise au seul contrôle visé par la partie IV ». Les premiers commentateurs avertis de cette convention du Conseil de l'Europe ont estimé, sans doute sur la foi des travaux préparatoires, que cette disposition avait été introduite précisément «pour exclure toute possibilité pour les intéressés de se prévaloir directement devant les autorités ou devant les juridictions nationales des dispositions de la Charte ${ }^{23}$. Mais on sait aussi que cela n'a pas empêché certaines juridictions internes d'en faire application $^{24}$. L'exemple le plus connu est celui de la Cour suprême des Pays Bas, laquelle a admis à plusieurs reprises l'effet direct des articles 6 par. 4 et 31 de la Charte relatifs au droit de grève et aux restrictions et limitations dont peuvent faire l'objet les droits consacrés par ce texte ${ }^{25}$. Cette jurisprudence est loin d'être isolée ${ }^{26}$.

Il ressort de ces éléments que la clause d'exclusion du contrôle juridictionnel que l'on rencontre parfois dans les instruments internationaux de protection des droits sociaux ${ }^{27}$ procède moins d'une impossibilité «naturelle » que de la réticence des États à assumer l'effectivité de leur protection. Elle fait d'ailleurs, selon la juste observation de Nicolas Valticos ${ }^{28}$, bon marché de l'autonomie des ordres juridiques nationaux. De fait, la « justiciabilité » des dispositions internationales relatives aux droits sociaux sera surtout fonction de l'orientation moniste ou dualiste de l'ordre juridique national considéré et des techniques juridiques mises en œuvre par les juges internes, lesquelles traduisent la détermination plus ou moins forte de ces juges à promouvoir la pleine jouissance des droits en question.

Reste évidemment un dernier argument de poids, lié au fait que la jouissance des droits sociaux, contrairement aux droits civils et politiques, requiert une intervention des pouvoirs publics. De cela, l'on déduit, à juste titre dans certains cas, que ces droits sont dépourvus d'effet direct et, à tort selon nous, qu'ils ne sont pas, en euxmêmes, susceptibles de protection juridictionnelle.

\section{Seconde proposition : la protection juridictionnelle de tous les droits sociaux est concevable si l'on admet qu'une telle protection ne se limite pas à la mise en œuvre judiciaire de droits directement applicables}

L'argument qui précède, outre qu’il occulte le fait que certains droits sociaux sont aussi directement applicables $^{29}$, ne pourrait être admis qu'au prix d'une représentation à la fois tronquée et atrophiée du concept de «justiciabilité».

Cette représentation a ceci de tronqué qu'elle implique, dès lors qu'il s'agit de droits sociaux consacrés en la forme d'objectifs ou de principes, que l'exigence de protection juridictionnelle s'efface devant la puissance législative, laquelle est censée définir discrétionnairement les conditions de mise en œuvre de ces droits ${ }^{30}$. Il est à noter que ces conceptions ne sont pas qu'un souvenir du passé. Elles se sont encore illustrées récemment, avec force, à travers la Charte des droits fondamentaux de l'Union européenne. C'est ainsi que la plupart des «droits de solidarité » prévus par ce texte doivent s'exercer « conformément au droit communautaire et aux législations et pratiques nationales ", "dans les cas et conditions prévus par le droit communautaire et les législations et pratiques nationales » ou encore "selon les modalités établies par le droit communautaire et les législations et pratiques nationales ${ }^{31}$. Autant dire que le contenu de ces « droits » et leurs exigences sont laissés à la libre appréciation du législateur communautaire et / ou national. Au plan des principes, une telle situation n'est guère satisfaisante au regard de la théorie de l'État de droit, de même qu'elle n'est pas compatible avec le caractère de droits fondamentaux des droits sociaux, lequel impliquerait que ceux-ci s'imposent à tous les pouvoirs publics, $y$ compris au législateur, et qu'ils soient assortis d'une sanction constitutionnelle. Elle ne correspond d'ailleurs

23. P. Laroque, «La Charte sociale européenne», Droit social, $\mathrm{n}^{\circ}$ 3, 1979, p. 108.

24. Sur la position de la Charte sociale européenne dans les ordres juridiques des États parties, voir M. Bonnechère, "Charte sociale et droits nationaux", in La Charte sociale européenne, p. 105 sq. Voir aussi J.-F. Akandji-Kombé, L'Interaction normative entre l'Union européenne et le Conseil de l'Europe en matière de droits sociaux, publié par le Conseil de l'Europe, 1999.

25. Hoge Raad, 30 mai 1986, Nederlandse Jurisprudentie, 1986.

26. Voir J.-F. Akandji-Kombé, L'Interaction normative... ; ainsi que C. Sciotti, « L'applicabilité de la Charte sociale européenne dans l'ordre juridique des États contractants", in Droits sociaux et Droit européen, bilan et prospective de la protection normative, J.-F. Flauss (dir.), Bruxelles, Bruylant, 2003, p. 175 sq.

27. On notera que lors de l'élaboration du PIDESC, des tentatives avaient été faites pour y introduire une disposition précisant que ces dispositions sont «non applicables de manière directe». Elles ont en définitive échoué. Voir Comité des droits économiques, sociaux et culturels, observation nº 9 , précitée, par. 11.

28. Cité par M. Bonnechère, «Charte sociale... », p. 116.

29. Voir infra.

30. Rappelons que selon la doctrine encore majoritaire, représentée avec autorité par le Professeur J. Rivéro, «les droits de créance (dont la plupart des droits sociaux) ne peuvent recevoir satisfaction qu'après la mise en place d'un appareil destiné à répondre aux exigences des particuliers. Le service public est donc, pour la satisfaction de tels droits, le procédé le plus normal. Tant que le service n'est pas créé, tant que l'État n'a pas réuni les moyens nécessaires pour s'acquitter de son obligation, le droit du créancier ne peut s'exercer. Or, il ne va pas, et ne peut aller pratiquement, jusqu'à contraindre l'État à créer le service nécessaire [...] ». Et l'auteur de conclure que «la satisfaction des droits de créance laisse donc à l’État un pouvoir d'appréciation discrétionnaire extrêmement large, de telle sorte que l'objet du droit reste pratiquement indéfini jusqu'à ce que le législateur ait procédé aux choix nécessaires». Voir J. Rivéro, Libertés publiques, vol. 1 : Les Droits de l’homme, Paris, PUF, 1974, p. 100 sq.

31. C'est le cas en ce qui concerne le droit à l'information et à la consultation des travailleurs au sein de l'entreprise (art. 27), le droit de négociation et d'action collectives (art. 28), le droit à la protection en cas de licenciement injustifié (art. 30), le droit à la sécurité sociale et à l'aide sociale (art. 34), le droit à la protection de la santé (art. 35), ainsi que le droit d'accès aux services d'intérêt économique général (art. 36). 
pas davantage à l'état actuel du droit constitutionnel de la grande majorité des États européens ${ }^{32}$, les droits sociaux étant consacrés au minimum comme principes ou objectifs à valeur constitutionnelle, constituant en tant que tels des normes du contrôle de constitutionnalité des lois. Le cas français est à cet égard assez représentatif. On sait que le Conseil constitutionnel, dans sa fameuse décision du 16 juillet 1971 relative au droit d'association, a intégré au bloc de constitutionnalité le préambule de la Constitution de 1946, lequel proclame notamment certains principes politiques, économiques et sociaux " particulièrement nécessaires à notre temps » ${ }^{33}$. Ces principes ont dès lors acquis, de l'avis général, valeur constitutionnelle dans leur ensemble ${ }^{34}$. Or, outre le contrôle qu'opère la juridiction constitutionnelle sur le respect par le législateur des exigences constitutionnelles en matière sociale ${ }^{35}$, et qui manifeste de l'obligation pour le Parlement de respecter les principes et objectifs sociaux affirmés par le préambule, il ressort assez clairement de la jurisprudence du Conseil que les pouvoirs publics sont liés par une obligation constitutionnelle d'action positive pour la réalisation des objectifs ou des principes contenus dans le préambule de la Constitution de 1946. C'est notamment le cas en matière de création des services publics, dont on sait la grande part qu'ils prennent dans la réalisation des droits sociaux. En la matière, le Conseil a, en particulier dans la décision $n^{0}$ 86-207 DC des 25-26 juin 1986 dite "privatisations », indiqué que certains de ces services étaient " exigés par la Constitution ${ }^{36}$, formule qui établit sans équivoque l'existence, dans le chef du législateur et du pouvoir réglementaire, d'une obligation constitutionnelle impérative tenant à leur mise en place $^{37}$. Selon les commentateurs, il en irait ainsi notamment des services de santé, de l'enseignement, de l'aide sociale, ou encore de l'emploi ${ }^{38}$. Sans doute l'affirmation de l'obligation reste-t-elle, dans le contexte juridique français, largement théorique en l'absence de possibilités d'action en inconstitutionnalité pour omission législative $^{39}$. Elle ne laisse pas moins entrevoir objectivement la possibilité d'un contrôle juridictionnel. Il est à noter qu'un tel contrôle est, au demeurant, déjà mis en œuvre au plan international par la Cour $\mathrm{EDH}$, particulièrement lorsque celle-ci apprécie le respect par les États parties des obligations positives impliquées par les dispositions de la Convention EDH, obligations qui s'apparentent dans une large mesure à ce que l'on rencontre dans les conventions internationales de protection des droits sociaux. Sans doute la Cour s'est-elle jusqu'à présent montré plutôt frileuse en ce qui concerne à la fois l'étendue et le contrôle de ces obligations. Sa jurisprudence n'en montre pas moins que la différence entre ce type d'obligations et les obligations négatives ou de non-ingérence tient moins au mode de contrôle qu'à son intensité.

L'argument selon lequel les droits sociaux ne seraient pas d'effet direct et, par conséquent, seraient non susceptibles de protection juridictionnelle doit être rejeté aussi parce qu'il suppose que la «justiciabilité » soit comprise exclusivement comme l'action du juge tendant à la sauvegarde de droits subjectifs contre l'emprise ou l'ingérence d'autrui, personne publique ou privée.

Une telle conception de la «justiciabilité » n'est plus défendable en l'état actuel du droit, et notamment du droit européen. Un détour par le droit de l'Union européenne suffit à montrer combien la protection juridictionnelle des personnes s'est enrichie dans ses modalités. Sur ce point, deux apports majeurs de la jurisprudence de la Cour de justice des Communautés relative à l'effet direct méritent d'être rappelés. Sur un plan général, l'arrêt de principe en la matière, Van Gend et Loos, rendu le 5 février $1963^{40}$, a eu le mérite de montrer qu'il n'y a pas de frontière rigide entre droits des particuliers et obligations de l'État. Des secondes l'on peut déduire les premiers, pour peu que la disposition considérée soit précise et inconditionnelle ${ }^{41}$. L'autre apport se situe sur le terrain

32. Voir C. Grewe, «Les droits sociaux constitutionnels, propos comparatifs à l'aube de la Charte des droits fondamentaux de l'Union européenne», Revue universelle des droits de l'homme, vol. 12, $\mathrm{n}^{\circ}$ 3-5, p. 85 sq. Voir F. Dreyfus, «Les droits économiques et sociaux dans quelques Constitutions récentes ", in L'État de droit. Mélanges en l'honneur de Guy Braibant, Paris, Dalloz, 1996, où l'auteur esquisse une comparaison entre la Constitution sud-africaine et les Constitutions des États d'Europe centrale et orientale, constatant le renouveau constitutionnel des droits sociaux fondamentaux.

33. Sur la teneur et les implications juridiques de ces principes, voir Le Préambule de la Constitution de 1946. Histoire, analyse et commentaires, G. Conac, X. Prétot et G. Teboul (dir.), Paris, Dalloz, 2001, notamment le commentaire des alinéas 2 (principes particulièrement nécessaires à notre temps : G. Teboul, p. 59 sq.), 5 (droit au travail et à l'emploi: M. Borgetto, p. 127 sq.), 11 (droit à la santé et protection sociale: X. Prétot, p. 261 sq.), et 13 (droit à l'instruction, à la formation et à la culture: J.-P. Costa, p. 313 sq.).

34. Comme le note le Professeur F. Luchaire après inventaire, seuls les trois derniers alinéas du préambule, relatifs à la défunte Union française, n'ont manifestement pas valeur constitutionnelle. Voir Le Conseil constitutionnel, t. I : Organisation et Attributions, $2^{\mathrm{e}}$ éd., Paris, Economica, $1997, \mathrm{p} .167$.

35. Voir par exemple la décision 401 DC du 10 juin 1998 relative à la loi sur la réduction du temps de travail, JORF, p. 9033 , où le Conseil rappelle le principe selon lequel le législateur doit respecter «les principes et règles de valeur constitutionnelle en ce qui concerne en particulier les droits et libertés fondamentaux reconnus aux employeurs et aux salariés».

36. Formule reprise dans la décision 232 DC du 7 janvier 1988.

37. Il est à noter que cette obligation n'existe pas en ce qui concerne les autres services publics. Le Conseil a en effet jugé dans la décision «privatisations» des 25-26 juin que «si la nécessité de certains services publics nationaux découle de principes ou de règles de valeur constitutionnelle, la détermination des autres activités qui doivent être érigées en service public national est laissée à l'appréciation du législateur ou de l'autorité réglementaire selon le cas».

38. Voir notamment en ce sens, F. Luchaire, La Protection constitutionnelle des droits et des libertés, Paris, Economica, 1987, p. 322 ; voir aussi, pour des aspects particuliers, J. Moreau, «Le droit à la santé», AJDA, juillet-août 1998, $\mathrm{n}^{\circ}$ spécial, Les Droits fondamentaux, une nouvelle catégorie juridique?, p. 188 ; R. Schwartz, «Éducation, une confluence des libertés publiques», ibid., p. 177.

39. Action que ne prévoit pas la Constitution française mais qu'institue par exemple la Constitution portugaise (article 283).

40. Aff. $26 / 62$, Rec. 1 .

41. Rappelons que dans cette affaire, la Cour de justice a déduit de l'article 12 du traité CEE aux termes duquel «les États membres s'abstiennent d'introduire entre eux de nouveaux droits de douane [...] et d'augmenter ceux qu'ils appliquent dans leurs relations commerciales mutuelles» le droit des particuliers au gel des droits de douane pendant la période de transition. La Cour juge que les droits des particuliers «naissent non seulement lorsqu'une 
de l'effet direct des directives ${ }^{42}$, où la Cour a progressivement abandonné l'approche en terme d'effet direct au profit d'une démarche plus large fondée sur l'invocabilité juridictionnelle de la norme ou l'applicabilité juridictionnelle; ce qui lui a permis de couvrir les hypothèses où le juge est appelé à écarter l'application d'une norme nationale incompatible avec des dispositions d'une directive dépourvues d'effet direct ${ }^{43}$ ou encore celle dans laquelle, pour l'application de telles dispositions, il doit procéder à une interprétation conforme de la réglementation nationale ${ }^{44}$. En dehors des directives, la CJCE a même pu considérer que de simples recommandations pouvaient bénéficier d'un tel effet et est allée jusqu'à énoncer une obligation du juge en la matière ${ }^{45}$. Ces jurisprudences intéressent le présent propos non seulement en raison des solutions consacrées mais aussi et surtout parce que celles-ci concernent d'un côté des actes qui, tout en étant obligatoires, se bornent en principe à énoncer des objectifs (les directives) et, de l'autre, des actes qui ne lient pas et qui, par conséquent, ne sauraient affecter le patrimoine juridique des personnes. C'est dire que, à supposer que les dispositions énonçant les droits sociaux ne soient pas d'effet direct, voire qu'elles se bornent à fixer aux États des objectifs, cela ne devrait pas les priver de tout effet de droit. Cette dernière remarque nous amène à notre troisième proposition.

\section{Troisième proposition : en définitive les exigences des droits sociaux - dont dépend aussi la nature du contrôle qui sera mis en œuvre - n'apparaissent pas fondamentalement différentes de celles des droits civils et politiques}

Cela est communément admis s'agissant de certains droits sociaux, précisément de ceux qui constituent en réalité des "libertés économiques et sociales", c'est-àdire des pouvoirs d'autodétermination, tels la liberté syndicale, le droit de grève ou la liberté professionnelle. C'est le cas aussi du principe de non-discrimination qui a des implications fortes dans le domaine social. Nul ne doute encore que ces droits et principes relèvent de la même problématique que les libertés dites "classiques» et qu'ils doivent jouir de la même protection. Mais on peut aller plus loin encore et envisager l'hypothèse d'une indivisibilité des droits de l'homme quant à leurs implications ou, pour être précis, quant à leurs exigences. Cela est possible à condition évidemment de changer de point de vue; à condition d'envisager ces exigences non plus sous l'angle des facultés reconnues aux bénéficiaires, mais plutôt sous celui des obligations induites par les droits et, puisqu'on est dans le cadre international, des obligations des États. On notera incidemment que cette approche présente l'avantage de mieux rendre compte de la logique première du contrôle international en matière de droits de l'homme qui est d'être un contrôle du respect par les États des obligations assumées.

Or, force est de constater que la nature de ces obligations ne varie guère, dans son principe, d'un groupe de droits à l'autre. Il est en effet possible d'affirmer que dans un cas comme dans l'autre, l'État est lié soit par l'obligation de respecter le droit, soit par celle de le protéger, soit encore par le devoir de le mettre en œuvre, étant précisé que ces obligations peuvent être assumées ensemble à l'égard d'un même droit. L'obligation de respect, qui implique la non-ingérence de l'État dans l'exercice du droit, est assumée à l'égard des droits civils et politiques bien sûr, mais elle l'est aussi à l'égard des droits économiques, sociaux et culturels. Ainsi, sous réserve d'une appréciation du caractère justifié de l'ingérence et de la proportionnalité des mesures prises, le droit au logement pourrait être considéré comme violé dans de nombreux cas et notamment lorsque l'État procède à des expulsions forcées; le droit d'accès aux soins lorsque les pouvoirs publics décident par exemple de fermer un service d'urgence, etc. Il en irait de même de l'obligation de protection, laquelle commande à l'autorité publique de veiller à ce qu'un tiers ne porte pas atteinte à un droit protégé et, dans le cas où le droit est tout de même violé, à prendre les mesures nécessaires pour que cette violation soit sanctionnée. On rappellera que c'est précisément dans cette optique que se place pour l'essentiel la jurisprudence de la Cour EDH relative aux «obligations positives». Celle-ci se ramène en effet à imposer aux États parties à la Convention européenne «d'adopter des mesures raisonnables et adéquates pour protéger les droits de l'individu », qu'il s'agisse du droit à la vie, du droit à une vie privée et familiale normale ou encore du droit à la nondiscrimination dans la jouissance des droits. Enfin, l'obligation de mise en œuvre, c'est-à-dire l'obligation d'intervenir pour assurer la jouissance effective des droits, vaut évidemment pour les droits sociaux, mais aussi en ce qui concerne les droits civils et politiques.

Le respect de chacune de ces obligations peut à n'en pas douter donner lieu à contrôle juridictionnel et, spécialement, à un contrôle international de cette nature.

Sans doute objectera-t-on que, au vu des instruments européens en vigueur, ces obligations ne se trouvent pas

attribution explicite en est faite dans les traités, mais aussi en raison d'obligations que le traité impose d'une manière bien définie, tant aux particuliers qu'aux États membres et aux institutions communautaires ».

43. Voir D. Simon, La Directive européenne, Paris, Dalloz, 1997.

43. Ibid., p. 94 sq.

44. Pour la position de principe de la Cour : CJCE, 10 avril 1984, Von Colson et Kamann, aff. 14 / 83, Rec. 1891 ; 13 novembre 1990 , Marleasing, aff. C-106 / 89 , Rec. I-4135.

45. CJCE, 13 décembre 1989, Grimaldi, aff. 322 / 88, Rec. 4407. Dans cet arrêt, la Cour, tout en déniant aux recommandations un effet direct, pose l'obligation pour les juridictions nationales de les prendre en compte lorsqu'elles sont susceptibles d'éclairer l'interprétation d'autres dispositions de droit national ou de droit communautaire. 
distribuées de la même manière dans la Convention européenne des droits de l'homme et dans la Charte sociale européenne. Mais est-on absolument certain, à supposer cette objection fondée, qu'il ne s'agisse pas là d'un effet de la formulation des dispositions des traités considérés?

$\mathrm{Si}$, pour conclure, on admet que la protection juridictionnelle des droits sociaux est concevable dans son principe, il resterait encore à l'organiser. Et si l'on se réfere au cadre de la Convention $\mathrm{EDH}$, force est de constater que le contexte est relativement défavorable. En effet, la sauvegarde de l'efficacité du système conduit à rechercher les moyens de réduire la charge des affaires à juger par la Cour EDH plutôt qu'à multiplier les possibilités de saisine. Pour ce qui concerne les droits sociaux, on peut se demander s'il n'y a pas là une erreur stratégique. À l'heure où se développement des systèmes parallèles de protection, dans lesquels s'affirme l'indivisibilité des droits de l'homme, la Convention EDH et sa Cour ne devraient-elles pas plutôt "prendre la main ${ }^{46}$ ? Après tout, et pour reprendre l'interrogation du Secrétaire général du Conseil de l'Europe «l'Europe peut-elle continuer d'être crédible et se prétendre l'Europe des droits de l'homme tant que les questions sociales seront considérées en termes de problèmes sociaux, d'amalgames de difficultés et d'obstacles, et non pas en tant que caractéristiques naturelles de la vie de toute société, indissociablement liées à la dignité de chacun de ses membres » ${ }^{47}$ ? La réponse est à l'évidence «non ». Il faudrait en tirer les conséquences.

46. C'est ce que suggère le Professeur F. Sudre en même temps qu'il montre que tous les droits sociaux pourraient être protégés par la Cour à partir des dispositions actuelles de la Convention: «Exercice de "jurisprudence-fiction" : la protection des droits sociaux par la Cour européenne des droits de l'homme", in Les Droits sociaux..., p. 145 sq.

47. Document CM (2000) du 14 novembre 2000, p. 28. 
